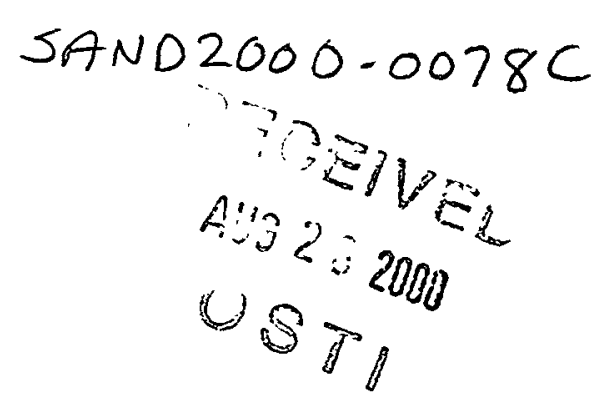

\title{
What Makes a Beam Shaping Problem Difficult
}

\author{
L.A. Romero *F.M. Dickey $\ddagger$
}

\begin{abstract}
We discuss what we believe are the three most important factors effecting the difficulty of a beam shaping problem: scaling, smoothness, and coherence.
\end{abstract}

\section{Introduction}

This paper is concerned with the question, "What makes a beam shaping problem difficult?" We believe this is the most fundamental question that needs to be answered if one is to understand the beam shaping process. Although the results summarized in this paper go a long way towards answering this question, they are by no means the final word on the subject.

If one has no idea how to answer this question, one can easily be deceived by the merits of an algorithm for designing a beam shaper. If a designer first encounters a problem that is very easy, they might conclude that their algorithm can successfully design any beam shaping system. On the other hand, if they first encounter a hard problem, they might conclude that their algorithm doesn't work on any beam shaping system. Furthermore, if you don't know what makes a beam shaping problem difficult, you have no idea how to modify your system so that the problem is easier.

In general terms, beam shaping is concerned with the problem of taking a beam with an irradiance distribution $I_{\text {in }}(x, y)$ and passing it through an optical system whose output gives us a beam with irradiance distribution $I_{\text {out }}(x, y)$ at some output plane. Ideally, we would like to have a lossless system where the energy of the input and output beams are identical. Assuming that we can apply the laws of geometrical optics, it is possible to design a lossless optical system that transforms any given irradiance distribution $I_{i n}(x, y)$ into any desired output $I_{\text {out }}(x, y)$, provided only that the total energy of the input and output beams are identical. However, when we take into account the full wave nature of the beam, it is no longer possible to do this in general (see ch. 2 in [1]).

\footnotetext{
- Sandia National Laboratories, Albuquerque N.M. 87185

†Sandia National Laboratories, Albuquerque N.M., 87185

† Sandia is a multiprogram laboratory operated by Sandia Corporation, a Lockheed Martin Company, for the United States Department of Energy under Contract DE-AC04-94AL85000
} 


\section{DISCLAIMER}

This report was prepared as an account of work sponsored by an agency of the United States Government. Neither the United States Government nor any agency thereof, nor any of their employees, make any warranty, express or implied, or assumes any legal liability or responsibility for the accuracy, completeness, or usefulness of any information, apparatus, product, or process disclosed, or represents that its use would not infringe privately owned rights. Reference herein to any specific commercial product, process, or service by trade name, trademark, manufacturer, or otherwise does not necessarily constitute or imply its endorsement, recommendation, or favoring by the United States Government or any agency thereof. The views and opinions of authors expressed herein do not necessarily state or reflect those of the United States Government or any agency thereof. 


\section{DISCLAIMER}

Portions of this document may be illegible in electronic image products. Images are produced from the best available original document. 
In [2] Dickey et al describe three basic approaches to designing beam shaping systems: apertured beam shapers, beam integrators, and field mappers. In an apertured beam shaping system one tries to achieve a uniform irradiance distribution for the output by blocking off all but a small portion of the input beam. Here we are making the assumption that the input aperture is close enough to the output plane that the field in the aperture is not significantly modified while propagating from the input aperture to the output plane. Systems of this sort have the significant disadvantage that they are not lossless.

In a beam integrator, one divides the input beam into a large number of facets, and tries to spread the energy in each facet over the whole output region so that it has the shape of the desired output irradiance distribution. The total field of the output beam is the sum of the fields from each of the individual facets. Systems of this kind exhibit undesirable interference effects when applied to coherent laser beams. However, as we will see, they work well for incoherent beams.

A field mapper is a system that takes into account the wave nature of the problem, and attempts to transform the field at the input plane so that it has the desired irradiance distribution at the output plane. In the geometrical optics limit, a field mapper maps a small portion of the input beam into a small portion of the output beam. Field mappers work well for coherent laser beams, but do not work well for incoherent beams.

As pointed out in [2], there are numerous ways of implementing these approaches, but all approaches seem to fall into one of these categories.

In this paper we concentrate on three aspects that are critical for determining if a beam shaping problem is hard.

- Scaling- How the wavelength, the size of the input and output beams, and the focal length of the system effect the difficulty of the problem.

- Smoothness- How discontinuities in the the input and the desired output irradiance distributions effect the difficulty of the problem.

- Coherence- How the coherence width of the laser effects the difficulty of the problem.

\section{Scaling}

In order to understand the effects of scaling on beam shaping we consider a specific example of a beam shaping system (Fig 1). We suppose that a collimated, coherent beam enters the input aperture, where it encounters a Fourier transform lens, and a beam shaping lens. The Fourier transform lens modifies the phase of the incoming beam by a quadratic phase factor, and the beam shaping lens modifies the phase by a factor that we would like to determine. Depending on the specific application it can be desirable to combine the two lenses into a single lens, or keep them separate. We now evaluate the irradiance at the focal plane of the transform lens. 


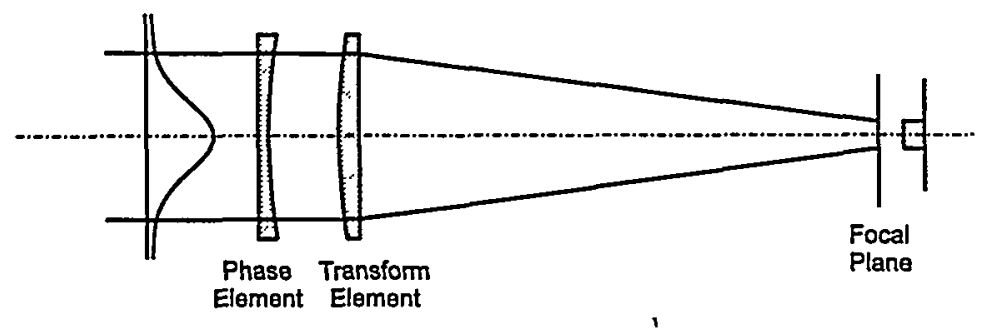

Figure 1: Schematic of a simple beam shaping system. A coherent collimated beam first goes through a phase element, then a transform lens. We are interested in the irradiance distribution at the focal plane. (Figure from [1])

To be more specific, we will assume that the input irradiance can be written as

$$
I_{i n}(x, y)=g^{2}(x / R, y / R)
$$

and the desired output irradiance can be written as

$$
I_{\text {out }}(x, y)=Q(x / D, y / D)
$$

Note that we have used the notation $g(x / R, y / R)$ and $Q(x / D, y / D)$ rather than $g(x, y)$ and $Q(x, y)$ for the irradiance distributions. This allows us to consider families of beam shaping problems where the shape of the input and output stay the same, but their characteristic length scales change. We can then analyze the effect of changing the length scales in our problem.

Our approach to analyzing the scaling properties of beam shaping systems is motivated by the commonly used practice in fluid dynamics of using dimensionless equations and dimensionless numbers [6]. For example, when fluid flows past a sphere, the behavior of the flow depends on the Reynolds number

$$
R e=\frac{U_{0} \rho R}{\mu}
$$

where $U_{0}$ is the velocity far from the sphere, $R$ is the radius of the sphere, $\mu$ is the dynamic viscosity, and $\rho$ is the density. If two flow have the same Reynolds number, the patterns of the flow fields will be identical, after rescaling our coordinates. However, if the Reynolds numbers are different, the flow patterns can look dramatically different. For example, in one case the flow could be turbulent, and in the other case not.

A similar situation occurs when we apply Fresnel difraction theory to analyzing the beam shaping problem. We will write down the dimensionless beam shaping equations for a separable beam shaping system, that is one where $g(\xi, \eta)=g_{1}(\xi) g_{2}(\eta)$, and $Q_{1}\left(\alpha_{1}, \alpha_{2}\right)=Q_{1}\left(\alpha_{1}\right) Q_{2}\left(\alpha_{2}\right)$. The problem of turning a circular Gaussian into a square flat-top beam is an example of a separable problem. A separable problem can be divided up into two one dimensional 
beam shaping problems. The dimensionless beam shaping problem can be written as follows. For given shape functions $g(\xi)$ and $Q(\alpha)$ (we have dropped the subscripts), and for a given value of

$$
\beta=\frac{2 \pi R D}{\lambda f}
$$

try to find a phase function $\phi(\xi)$ and a constant ' $A$ such that

$$
G(\omega)=\int_{-\infty}^{\infty} g(\xi) e^{-i \omega \xi} e^{i \beta \phi(\xi)} d \xi
$$

satisfies

$$
\mid G\left(\left.\omega\right|^{2}=A \frac{2 \pi}{\beta} Q(\omega / \beta)\right.
$$

In our definition of the parameter $\beta, R$ is the characteristic length of the input beam, $D$ is the characteristic length of the output beam, $f$ is focal length of the Fourier transform lens, and $\lambda$ is the wavelength of light. In these equations eqn. (1) is the dimensionless expression of the fact that the field distribution at the focal plane is the Fourier transform (times a phase factor that is not important if we are only interested in the irradiance) of the field at the aperture modified by the phase function $\phi(\xi)([3])$. The equation (2) is the dimensionless statement of the fact that we would like the irradiance at the focal plane to have the desired distribution $Q(\alpha)$.

Note that once we are given the functions $g(\xi)$ and $Q(\alpha)$, the dimensionless beam shaping equations depend on only one parameter, $\beta$. If we have two beam shaping systems that have the same phase functions $\phi(\xi)$, and input fields $g(\xi)$, and the same value of $\beta$ they will both exhibit identical irradiance distributions at the focal plane after merely rescaling our axes.

It is well known that the geometrical optics limit is a small wavelength approximation. The parameter $\beta$ is a measure of how well the geometrical optics limits applies. If $\beta$ is large, a system designed using geometrical optics will work well. However, if $\beta$ is small such a system will not work well. In fact , suppose we could solve the beam shaping problem exactly. the uncertainty principle from signal analysis [4] shows that we must have

$$
\beta^{2} \Delta_{g}^{2} \Delta_{Q}^{2} \geq 1 / 4
$$

where

$$
\begin{aligned}
\Delta_{g}^{2} & =\frac{\int_{-\infty}^{\infty} \xi^{2} g^{2}(\xi) d \xi}{\int_{-\infty}^{\infty} g^{2}(\xi) d \xi} \\
\Delta_{Q}^{2} & =\frac{\int_{-\infty}^{\infty} \alpha^{2} Q(\alpha) d \alpha}{\int_{-\infty}^{\infty} Q(\alpha) d \alpha}
\end{aligned}
$$

Since $\Delta_{g}$ and $\Delta_{q}$ are independent of $\beta$, this shows that if $\beta$ is too small, we cannot do a good job of beam shaping. 


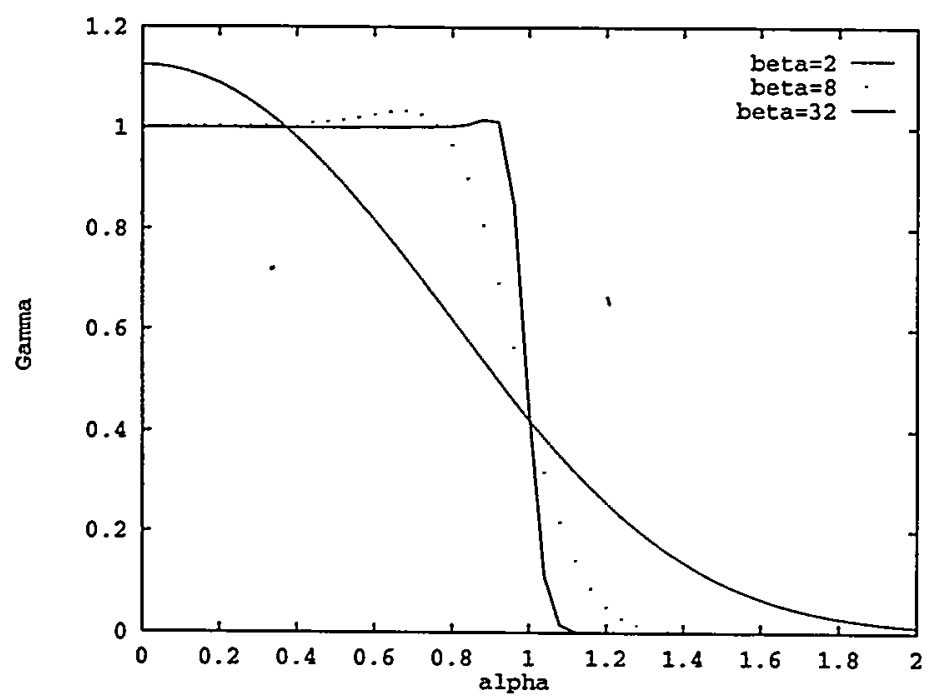

Figure 2: The effect of the parameter $\beta$ on the problem of turning a Gaussian into a flat-top. In this plot $\alpha=\omega / \beta$, and $\Gamma=\frac{\beta}{2 \pi A}|G(\omega)|^{2}$. (Figure from ([?]))

If the value of the parameter $\beta$ is large, we can use the method of stationary phase [5] to approximate the integral in (1) . It can be shown that the leading order term in the method of stationary phase is identical to the geometrical optics approximation. We can use the stationary phase approximation, or equivalently Fermat's principle to show that the phase $\phi(\xi)$ must satisfy the following set of equations.

$$
\begin{gathered}
\frac{d \phi}{d \xi}=\alpha(\xi) \\
\int_{-\infty}^{\xi} g^{2}(s) d s=A \int_{-\infty}^{\alpha(\xi)} Q(s) d s
\end{gathered}
$$

Here $A$ is a constant chosen so that the total energy of the input and output beams are the same.

$$
\int_{-\infty}^{\infty} g^{2}(s) d s=A \int_{-\infty}^{\infty} Q(s) d s
$$

and

$$
\alpha=\frac{\omega}{\beta}
$$

Taking the derivative of this equation we find that

$$
g^{2}(\xi)=A Q(\alpha(\xi)) \frac{d \alpha}{d \xi}
$$




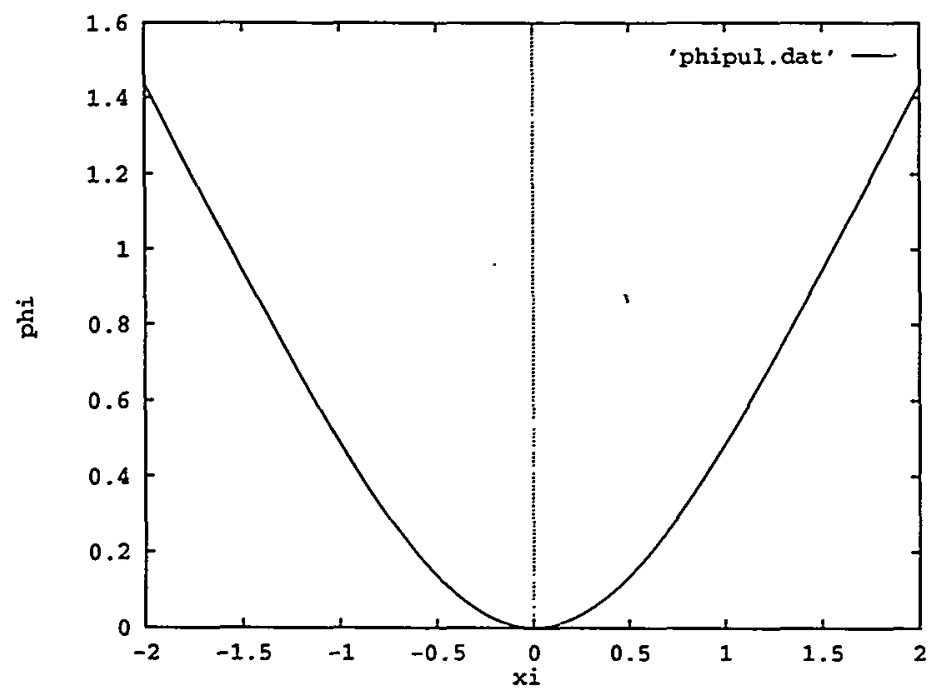

Figure 3: The phase function $\phi(\xi)$ for turning a Gaussian into a flat-top beam. Note that even though $Q(\alpha)$ is discontinuous, the phase function is infinitely differentiable.

Once we have determined the constant $A$, the equations (3) and (6) are a system of differential equations that allow us to determine the functions $\alpha(\xi)$ and $\phi(\xi)$. These equations determine the phase functions $\phi(\xi)$ in the geometrical optics limit. This phase function will accomplish the desired beam shaping if the parameter $\beta$ is sufficiently large. Figure 2) shows how the phase function designed using geometrical optics works for the problem of turning a Gaussian into a flat-top. When $\beta=2$, the output does not look much like a flat-top, but by the time $\beta=32$ the output is looking quite sharp. In figure 3) we show the phase function that we use for turning the Gaussian into the flat-top.

The designer of a beam shaping system can influence the parameter $\beta$ in several different ways. As one example, it is possible to expand the beam using a telescope before putting it into our simplified beam shaping system. This allows us to increase the value of the parameter $R$, and hence increase $\beta$. This technique has been successfully employed by Dickey and Holswade (see ch 3 from [1])..

Although the results we have described were derived assuming that we had a coherent beam, they can also be useful in the design of incoherent beam shaping systems. Dickey and Holswade (ch 7 [1]) have shown that these results apply for multi-faceted beam shaping systems for incoherent beams. In particular, if the facets are smaller than the coherence width of the laser, then we can assume that the beam is coherent over each facet. We can now apply the results for coherent beams, where the characteristic dimension $R$ is not the dimension of the full aperture, but of each individual facet. 


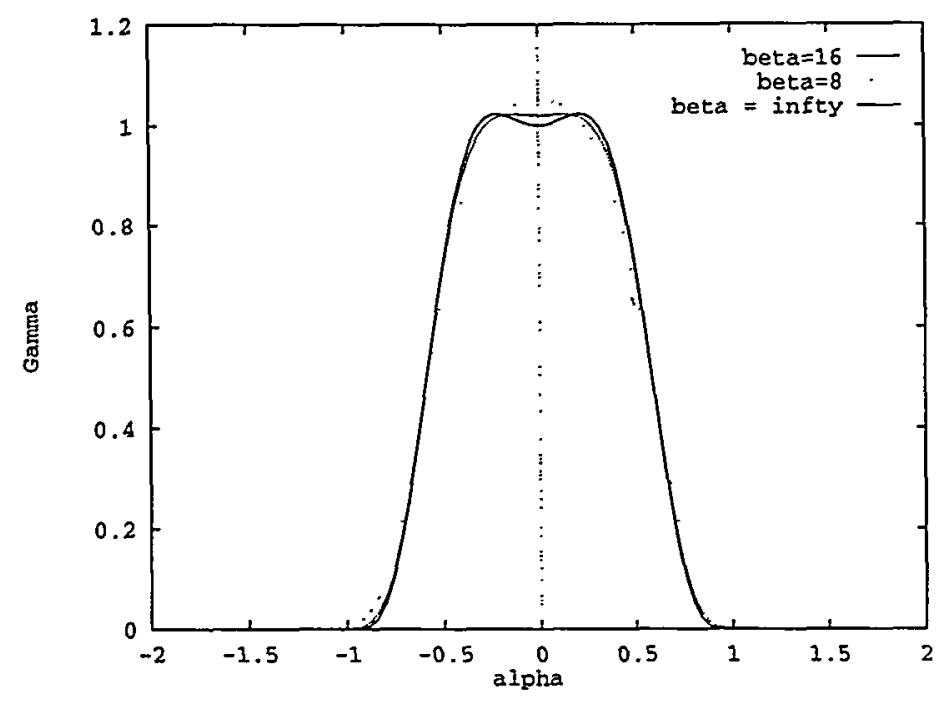

Figure 4: The effect of $\beta$ on the problem of turning a Gaussian into the profile $Q(\alpha)=\left(1+3 \alpha^{2}\right)\left(1-\alpha^{2}\right)^{5}$ for $|\alpha|<1$, and $Q=0$ for $|\alpha|>1$.

\section{Smoothness}

Although the size of the parameter $\beta$ mentioned in the last section is a crucial element in determining the difficulty of a beam shaping problem, it is by no means the only factor. If we once again restrict ourselves to problems where the incoming beam is coherent, the next most important factor influencing the difficulty of a beam shaping problem is the smoothness of the input and output beams.

In this section we show that some beam shaping problems require a much larger value of $\beta$ in order for a lens designed using geometrical optics to work well. In order to see why this is so we need to examine the errors obtained in making the geometrical optics approximation. This is equivalent to finding the higher order terms in the stationary phase approximation. We will see that discontinuities can slow down the convergence of the method of stationary phase.

The effect of discontinuities on the convergence of the method of stationary phase is similar to the effect of discontinuities on the convergence of Fourier series. If a periodic function has a discontinuity, the coefficients in the Fourier series die down like $1 / N$, where $N$ is the number of Fourier components. If the function is continuous, but has a discontinuity in its first derivative, the coefficients die down like $1 / N^{2}$; and in general if the first $k-1$ derivatives are continuous but it has a discontinuity in its $k$ th derivative, the coefficients will die like $1 / N^{k+1}$. If all of the derivatives are continuous, the coefficients will die down exponentially fast. It follows that the smoother a function is, the less 


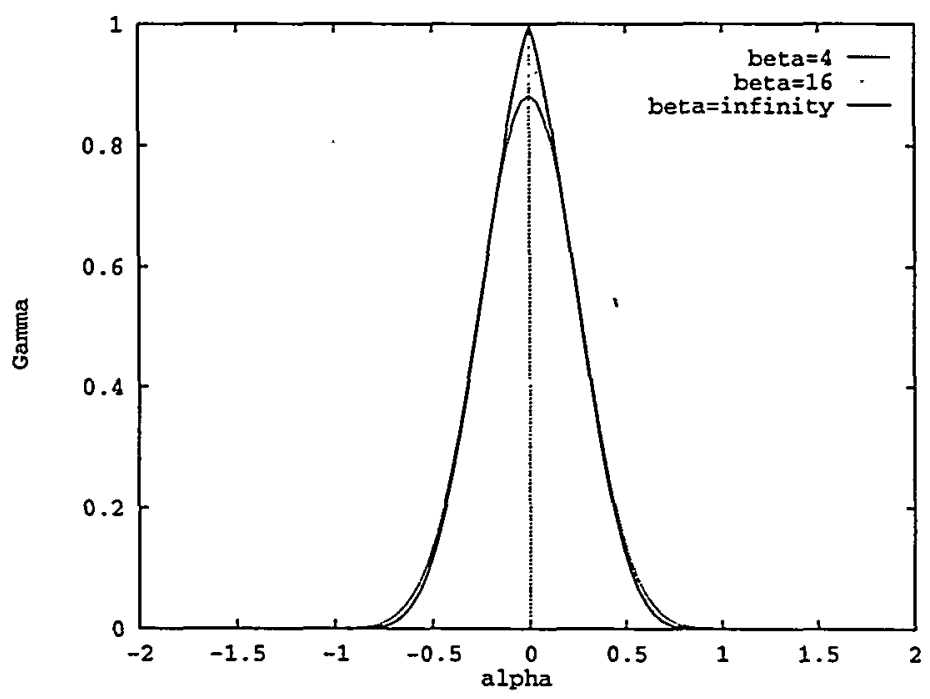

Figure 5: The effect of $\beta$ on the problem of turning a Gaussian into the profile $Q(\alpha)=(1-|\alpha|)\left(1-\alpha^{2}\right)^{5}$ for $|\alpha|<1$, and $Q=0$ for $|\alpha|>1$. Note that we get much slower convergence near the discontinuity in the derivative of $Q$.

Fourier components we need in order to approximate it well.

We have a somewhat similar situation for the beam shaping problem. However, there are some significant differences that can be misleading if one carries this analogy too far. When making this analogy, it is best to concentrate on the continuity of the phase function $\phi(\xi)$ of the beam shaping lens rather than on the continuity of the desired output. Once we understand how the continuity of $\phi$ effects the convergence with $\beta$, we can then understand how the continuity of $Q(\alpha)$ effects the continuity of $\phi(\xi)$.

We begin by supposing we have a beam shaping lens that has a shape $\phi(\xi)$, and that the field at the aperture has the distribution $g(\xi)$. In our discussion we will assume that $g(\xi)$ is sufficiently smooth. Suppose that geometrical optics predicts that this lens transforms the initial irradiance $g^{2}(\xi)$ into $Q(\alpha)$ at the output plane. How does the actual irradiance distribution created by this lens differ from the distribution predicted by geometrical optics? We can answer this question by considering the next order term in the method of stationary phase. If the lens has continuous derivatives up to third order, the two results will have a relative error of order $1 / \beta^{2}$. If the lens has discontinuity in the third derivative, the relative error will only die down like $1 / \sqrt{\beta}$. If the phase function has a discontinuity in its second derivative, we end up with a situation that is similar to diffraction by a straight edge. In this case the convergence will depend on how close we are to the point of discontinuity. As we approach the discontinuity, the convergence is extremely slow. The convergence is even worse if the phase function has a discontinuity in its first derivative. 
We see that as $\phi$ becomes less smooth, the convergence becomes slower. There is one difference between this case and our example of the decay of the terms in a Fourier series. In that case we kept getting better convergence as more and more derivatives became continuous. However, for the beam shaping problem, we do not get significantly better convergence when we have 4 continuous derivatives than when we have just three continuous derivatives.

Now that we know how the continuity of $\phi(\xi)$ effects the convergence towards the geometrcial optics limit, we consider how the continuity of $Q(\alpha)$ effects the continuity of $\phi(\xi)$. In order to understand this we must examine the equations governing one dimensional geometrical beam shaping (3),(6). The equation 3 shows that if $\alpha$ has a discontinuity in the kth derivative, then $\phi(\xi)$ has a discontinuity in the $k+1$ st derivative.

Suppose the function $Q(\alpha)$ has a discontinuity at $\alpha_{0}$. Suppose we are solving our differential equations for $\alpha(\xi)$ and $\phi(\xi)$, and that at $\xi_{0}$ we have $\alpha\left(\xi_{0}\right)=\alpha_{0}$. If $g\left(\xi_{0}\right) \neq 0$, the equation 6 implies that there will be a discontinuity in $\frac{d \alpha}{d \xi}$, and hence a discontinuity in the second derivative of $\phi$. This sort of reasoning shows that typically a discontinuity in the $k$ th derivative of $Q$ will lead to a discontinuity in the $k+2$ derivative of $\phi$. It appears that we can look at the continuity of $Q$ and determine how difficult the beam shaping problem is.

Figure 4) shows an example of the beam shaping problem where we attempt to turn a Gaussian beam into the function $Q(\alpha)-\left(1+3 \alpha^{2}\right)\left(1-\alpha^{2}\right)^{5}$ for $|\alpha|<1$, and $Q(\alpha)=0$ for $|\alpha|>1$. This function has been chosen so that the first five derivatives are continuous even at $\alpha= \pm 1$. We see that the convergence towards the geometrical optics limit is very fast. When $\beta=16$ we are already close to the geometrical optics limit.

Figure 5) shows an example of the beam shaping problem where we attempt to turn a Gaussian beam into the function $Q(\alpha)-(1-|\alpha|)\left(1-\alpha^{2}\right)^{5}$ for $|\alpha|<1$, and $Q(\alpha)=0$ for $|\alpha|>1$. This function has been chosen so that there is a discontinuity in the first derivative at $\alpha=0$, but the function is differentiable elsewhere. Note that we get slower convergence towards the geometrical optics limit near the point of discontinuity.

Figures 6) and 7) are examples where we attempt to turn a Gaussian input into a beam that has discontinuities in the output. In figure 6) $Q(\alpha)=3 / 4$ for $|\alpha|<1 / 2, Q(\alpha)=1$ for $1 / 2<|\alpha|<1$, and $Q=0$ for $|\alpha|>1$. In figure 7) we have $Q(\alpha)=0$ for $|\alpha|<1 / 2, Q(\alpha)=1$ for $1 / 2<|\alpha|<1$, and $Q=0$ for $|\alpha|>1$. The phase function $\phi(\xi)$ for the profiles in figure 6 ) has a discontinuity in the second derivative, while the phase function for figure 7) has a discontinuity in the first derivative. Clearly the convergence towards the geometrical optics limit is bad in figure 6) and even worse in figure 7).

It is interesting to contrast figures 6) and 7) with figure 2). In all of these figures the function $Q(\alpha)$ has a discontinuity. However, the continuity of the phase function $\phi(\xi)$ used to generate these figures is much different. In figure 2 ) the phase function $\phi(\xi)$ is infinitely differentiable. This arises since as we integrate out differential equations of $\alpha(\xi)$ and $\phi(\xi)$, we have $g(\xi)=0$ at the point of discontinuity of $Q(\alpha(\xi))$. This situation arises when the discontinuity in $Q(\alpha)$ is at the end of its interval of definition. We see that discontinuities at 


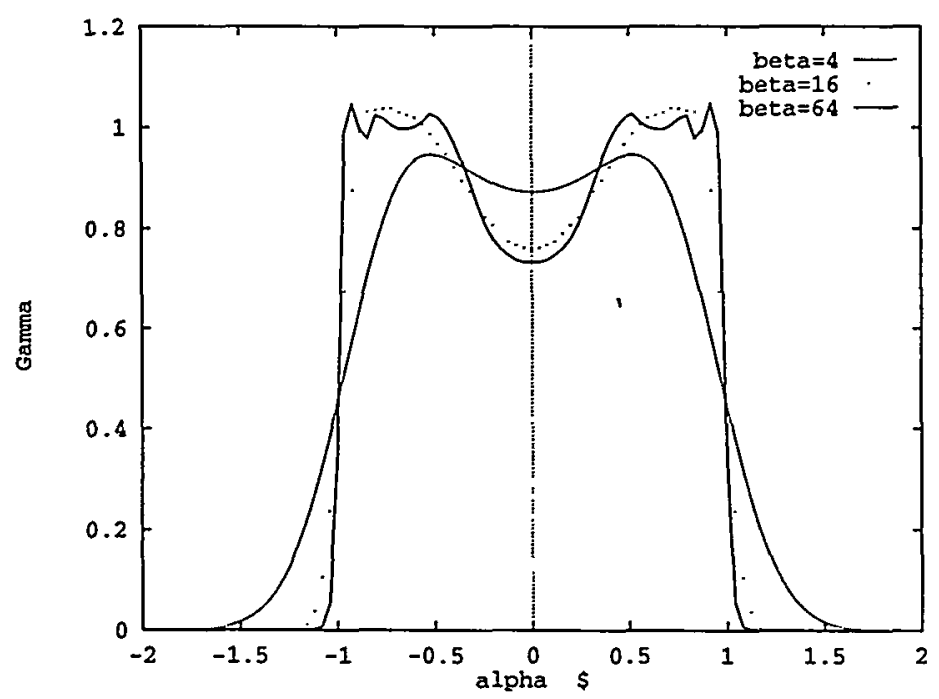

Figure 6: The effect of $\beta$ for the problem of turning a Gaussian into the profile $Q(\alpha)=3 / 4$ for $|\alpha|<1 / 2, Q(\alpha)=1$ for $1 / 2<|\alpha|<1$, and $Q=0$ for $|\alpha|>1$. (Figure from [1])

interior points are much more severe then discontinuities at the extremities.

\section{Coherence}

We once again analyze the situation where we pass a beam through an aperture that contains a Fourier transform lens, and a beam shaping lens; and we evaluate the irradiance at the output plane. However, we now drop the assumption that the laser is spatially coherent. Suppose we take a system that is designed using geometrical optics, and we pass a partially coherent beam of light through it. Unlike the coherent case, we cannot determine the irradiance at the output plane given the irradiance at the input plane. In order to determine the irradiance at the output, we must know the mutual intensity function $J\left(x_{1}, y_{1} ; x_{2}, y_{2}\right)$ at the input. For a given plane $z=$ const, this gives the correlation between the field at $\left(x_{1}, y_{1}\right)$ and at $\left(x_{2}, y_{2}\right)$. The function $J\left(x_{1}, y_{1} ; x_{1}, y_{1}\right)$ is the irradiance at $\left(x_{1}, y_{1}\right)$. It can be shown [7] that the mutual intensity at the output plane is given by

$$
\begin{gathered}
J\left(x_{1}, y_{1} ; x_{2}, y_{2}\right)=C\left(x_{1}, y_{1}, x_{2}, y_{2}\right) \int_{-\infty}^{\infty} \int_{-\infty}^{\infty} \int_{-\infty}^{\infty} \int_{-\infty}^{\infty} B\left(\xi_{1}, \eta_{1} ; \xi_{2}, \eta_{2}\right) e^{\frac{2 i}{\lambda(}\left(\xi_{1} x_{1}+\eta_{1} y_{1}-\xi_{2} x_{2}-\eta_{2} y_{2}\right.} d \xi_{1} d \xi_{2} d \eta_{1} d \eta_{2} \\
B\left(\xi_{1}, \eta_{1} ; \xi_{2}, \eta_{2}\right)=J\left(\xi_{1}, \eta_{1} ; \xi_{2}, \eta_{2}\right) e^{i \phi\left(\xi_{1}, \eta_{1}\right)} e^{-i \phi\left(\xi_{2}, \eta_{2}\right)}
\end{gathered}
$$




$$
C\left(x_{1}, y_{1}, x_{2}, y_{2}\right)=e^{\frac{i}{\lambda f}\left(x_{1}^{2}+y_{1}^{2}-x_{2}^{2}-y_{2}^{2}\right)} \frac{1}{(\lambda f)^{2}}
$$

We would like to determine function $\phi(\xi, \eta)$ so that $J(x, y ; x, y)$ has the desired irradiance distribution. It is typical to assume that

$$
J\left(\xi_{1}, \eta_{1} ; \xi_{2}, \eta_{2}\right)=\sqrt{I\left(\xi_{1}, \eta_{1}\right) I\left(\xi_{2}, \eta_{2}\right)} \mu\left(\xi_{1}-\xi_{2}, \eta_{1}-\eta_{2}\right)
$$

Here $\mu$ is the complex coherent factor. The function $\mu$ tells how well the fields at $\left(\xi_{1}, \eta_{1}\right)$ and $\left(\xi_{2}, \eta_{2}\right)$ are correlated. If $\mu$ is constant, the beam is coherent, and if it is a delta function, the beam is totally incoherent.

In general this gives us a much more complicated beam shaping problem than in the coherent case. The major complication arises from the fact that we now need to evaluate a four dimensional integral rather than a two dimensional integral. When the function $\mu$ is constant, the four dimensional integral is separable, and we end up with an identical beam shaping problem as in the coherent case. Even when $\mu$ is not constant, we can apply the method of stationary phase to evaluate this integral. It is not much more difficult to apply the method of stationary phase to this 4 dimensional integral, than to the two dimensional integral that appears in the coherent case. When we apply the method of stationary phase to this integral we get exactly the same answer that we get in the coherent case. However, the method of stationary phase assumes that $\mu$ as well as $I(\xi, \eta)$ can be approximated as being constant in the vicinity of the stationary point. If the function $\mu$ is varying rapidly enough, this will not be a valid assumption. A detailed analysis of this situation shows that the geometrical optics approximation will be valid provided both the parameter $\beta$ and

$$
\beta^{\star}=\beta(l R)^{2}
$$

are large. Here $l$ is the coherence width of the incoming beam. This can be written as

$$
\beta^{\star}=\frac{D l^{2}}{2 \pi \lambda f R}
$$

One might have guessed that in the partially coherent case we should replace the size of the aperture $R$ in $\beta$ by the coherence width $l$. However, we have a more extreme result. We must replace $R$ by $l(l / R)$. This result shows that not only does it not do us any good to have an aperture that is significantly larger than the coherence width of the beam, it actually hurts us. Intuitively this result can be thought of as follows. In the partially coherent case, the lens can be thought of as being broken up into many lenses whose characteristic lengths are the coherence width of the laser beam. However, each of these small lenses maps a small part of the input beam onto a small part of the output beam. For this reason we are decreasing both $R$ and $D$ in the formula for $\beta$. This is why we get the factor $l(l / R)$ in the formula for $\beta^{\star}$.

When the incoming beam has a small coherence width relative to its width, it is desirable to use multi-faceted beam integrators to do beam shaping. In 


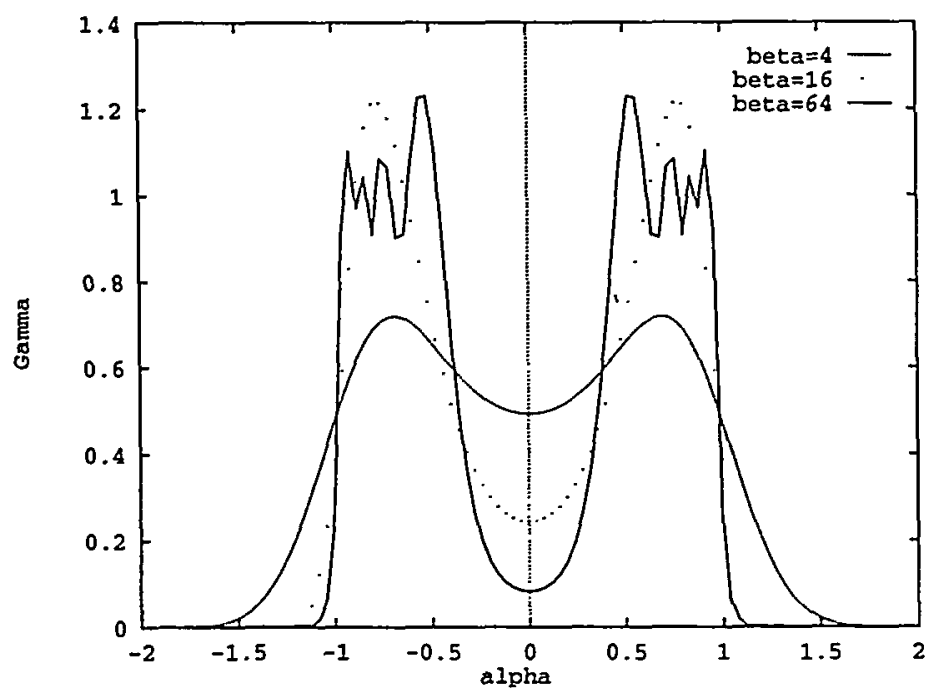

Figure 7: The effect of $\beta$ on the problem of turning a Gaussian into the profile with $Q(\alpha)=0$ for $|\alpha|<1 / 2, Q(\alpha)=1$ for $1 / 2<|\alpha|<1$, and $Q(\alpha)=0$ for $|\alpha|>1$. (Figure from [1])

this case we build a system of lenses so that each lens only sees a small portion of the beam. We try to make each facet small enough so that the incoming beam is nearly coherent over the whole area of the facet. We design each facet so that it spreads the input beam coming into the facet over the whole output plane. The resulting output will now be the sum of the output from each facet. Assuming the beam is completely incoherent, we will be able to add the irradiance distributions of each of the individiual facets. Since the beam is partially coherent, we will get some interference effects.

\section{Conclusions}

We have discussed the three factors that we believe are the most important in determining the difficulty of a beam shaping problem: scaling, smoothness, and coherence. Our arguments have been almost completely based on considering how these factors influence beam shaping lenses that were designed using geometrical optics. However, we believe that these factors control the difficulty of beam shaping problems even if one does not base ones design strategy on geometrical optics. For example, we have shown that a lens designed uisng geometrical optics will not work well unless $\beta$ is large, However, we have also shown that if $\beta$ is small the uncertainty principle shows that it is impossible to do a onnd inh of heam shaning nn matter how one desions ones lens

When the incoming beam has a small coherence width relative to its width, it is desirable to use multi-faceted beam integrators to do beam shaping. In 


\section{References}

[1] F.M. Dickey and S.C. Holswade, Laser Beam Shaping, Theory and Techniques, Marcel Dekker Inc, New York.

[2] F. M. Dickey, L.S. Weichman, R.N. Shagam, Laser Beam Shaping Techniques, SPIE proceedings, 4065 (2000).

[3] J.W. Goodman, An Introduction to Fourier Optics, New York, McGraw Hill, 1968 .

[4] L.E. Franks, Signal Theory, Englewood Cliffs, NJ; Prentice Hall, 1969.

[5] M.J. Lighthill, Waves in Fluids, Cambridge, Cambridge University Press , 1978.

[6] P.W. Bridgeman, Dimensional Analysis, New Have, Yale University Press, 1922.

[7] J.W. Goodman, Statistical Optics, New York, John Wiley, 1985. 